\title{
Les chaînes opératoires artistiques
}

\section{Thomas Golsenne}

\section{(2) OpenEdition \\ Journals}

Édition électronique

URL : https://journals.openedition.org/tc/7551

DOI : $10.4000 /$ tc. 7551

ISBN : 0248-6016

ISSN : 1952-420X

Éditeur

Éditions de l'EHESS

Édition imprimée

Date de publication : 24 décembre 2015

Pagination : 18-31

ISBN : 978-2-7132-2505-5

ISSN : 0248-6016

\section{Référence électronique}

Thomas Golsenne, « Les chaînes opératoires artistiques », Techniques \& Culture [En ligne], 64 | 2015, mis en ligne le 24 décembre 2018, consulté le 29 septembre 2022. URL : http:// journals.openedition.org/tc/7551 ; DOI : https://doi.org/10.4000/tc.7551 


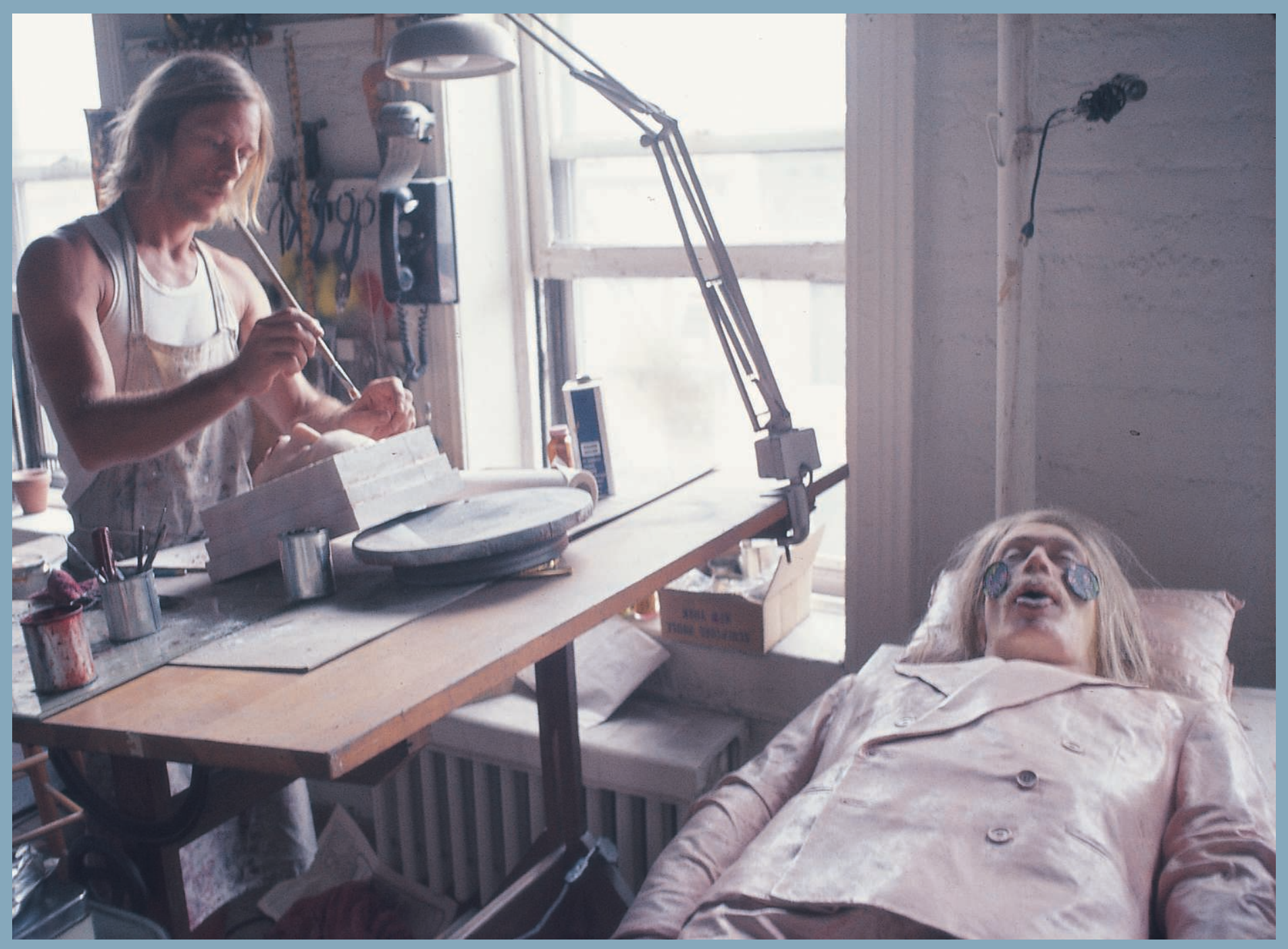




\section{Les chaînes opératoires artistiques}

Tout le monde le sait. L'art n'est pas la technique. Non, il vaut mieux dire : l'art hait la technique. Tim Ingold, qui a beaucoup écrit sur le sujet, résume parfaitement :

\footnotetext{
«Un objet ou une performance pourrait être une ceuvre d'art, plutôt qu'un simple artefact, à condition qu'il ou elle échapperait aux déterminations du système technologique, ou les transcenderait. Et son créateur pourrait être un artiste, plutôt qu'un simple artisan, du moment que l'œuvre serait comprise comme l'expression de son être subjectif. Là où les opérations technologiques sont prédéterminées, l'art est spontané ; où la manufacture d'artefacts est un processus de réplication mécanique, l'art est la production créative de nouveauté. »

« De fait, l'opposition entre art et technologie est, dans l'époque récente, devenue une part si établie de la pensée contemporaine sur la "condition humaine" que nous sommes enclins à l'utiliser comme une fenêtre à travers laquelle nous voyons les pratiques de tous genres, passées et présentes, occidentales et non-occidentales, humaines et animales. » (Ingold $2001: 18$, traduction de l'auteur)
}

On sait bien qu'il n'en a pas toujours été ainsi. On pense savoir que chez les Grecs, tekhnè signifiait toute activité de production d'artefacts, qu'ils soient utilitaires ou esthétiques ; on croit que ce mot a été traduit par les Romains par ars dans le sens d'activité nécessitant une habileté, et que ce sens a perduré jusqu'à l'expression « arts et métiers». On dit aussi qu'à la fin de l'Antiquité les auteurs ont séparé les arts mécaniques (arts manuels) des arts libéraux (arts intellectuels); qu'à partir du xve siècle en Italie, des intellectuels ont commencé à incorporer dans les arts libéraux des pratiques manuelles comme la peinture, l'architecture, et la sculpture (la peinture « cosa mentale » de Léonard de Vinci); qu’on a regroupé ces arts en « arts du dessin », jouant sur le double sens du mot en italien « disegno » (dessin/dessein); puis en « beaux-arts » ou « fine arts » au xviII siècle, moment où Ingold situe le divorce définitif entre l'art et la technique (il donne comme symptôme l'exclusion des graveurs de la Royal Academy of Fine Arts); puis, 
au bout du compte, on a forgé une catégorie conceptuelle unique, l'Art, au XIXe siècle, conçu notamment par Hegel comme activité de l'esprit qui se manifeste dans le sensible.

De ce point de vue, l'art du xxe siècle aurait accentué le divorce. Depuis Manet, le reproche adressé à l'art d'avant-garde par un public habitué aux critères traditionnels d'appréciation des œuvres d'art (le métier, le temps passé au travail, l'histoire représentée etc.) est toujours le même : " mon fils de cinq ans pourrait en faire autant ». Les artistes d'avant-garde eux-mêmes, en rejetant les enseignements académiques, en s'inspirant des « œuvres d'art nègre », comme on disait au début du xxe siècle, de l'art folklorique, des dessins d'enfants et de fous, ont prêté eux-mêmes le flanc à cette critique. Provoquer le hasard comme méthode de composition (Arp), utiliser des machines à produire des images comme l'appareil photographique (Man Ray), ou encore, dans le cas extrême de Duchamp, se contenter de poser sa signature ou un titre sur un objet acheté dans un grand magasin, furent autant de gestes d'artistes adressés contre l'habileté, contre le métier, bref, contre la technique. Un artiste new-yorkais des années 1960-1970, Paul Thek, le dit très clairement, au sujet de son propre travail, The Tomb (1967):

« Les œuvres corporelles avaient commencé à apparaître parce que je voulais arriver à comprendre comment faire un moulage entier de corps. Je n'avais jamais fait de moule ou de choses de ce type auparavant. Je travaillais avec un moulage de dentiste utilisé pour des plaies ouvertes et qui prend très rapidement. J'avais un atelier rempli de membres imparfaits recouverts de différentes cires colorées pour tester les teintes, c'était donc assez naturel de les utiliser. Si j’ai une esthétique, elle est enracinée en grande partie dans le pragmatisme. Si vous avez quelque chose sous la main, vous devez essayer d'en faire quelque chose. Je savais que la technique était sans importance. Dans un magazine de cette époque, quelqu'un [Pincus-Witten] me qualifia de maître-technicien et j'ai pensé, "C'est un non-sens absolu et une insulte : vous ne pouvez pas qualifier un artiste de maître-technicien — il s'agit de quelqu'un d'autre." » (Flood 1981 : 108)

On pourrait voir cette opposition féroce à la technique comme le renforcement de la dichotomie moderne entre conception et réalisation, la dimension artistique résidant entièrement dans la conception. C'est d'ailleurs à cette époque que se développe l'art conceptuel, dans l'héritage de Duchamp pour une part, et de la littérature d'autre part, où le lien traditionnel entre l'œuvre et l'artiste est complètement dissous. Lawrence Weiner, Sol LeWitt, ou Robert Filliou, avec son « principe d'équivalence » — bien fait, mal fait, pas fait — remirent en doute l'importance de la réalisation d'une pièce par l'artiste et l'importance de la qualité de l'exécution. Ce rejet découlait d'une éthique de l'économie, au sens où faire un objet (ce qu’on attend généralement des artistes) était considéré par les conceptuels comme un problème, voire comme une vanité malsaine dans un monde déjà saturé d'objets inutiles.

Cette histoire linéaire de la spiritualisation continue des arts en même temps que leur resserrement en un seul concept transcendantal est le récit habituel qui est énoncé à chaque fois qu'on veut expliquer pourquoi l'art est au-delà de la technique. Cela produit des effets sur les formes de connaissances : les historiens de l'art s'intéressent assez peu aux techniques employées par les artistes, laissant ce domaine aux restaurateurs. Comme le résume très bien Tim Ingold: 
selon l'opinion commune, la technique fonctionne, l'art signifie (Ingold 2001 : 19). Pour les historiens de l'art, qui cherchent à étudier la signification des œuvres d'art, il est donc assez légitime de laisser de côté le domaine des techniques. La question qu'ils adressent aux œuvres et aux artistes est : « Pourquoi ? », rarement « Comment ?».

Ce récit n'est pas entièrement faux, bien sûr. Mais il repose en grande partie sur une conception hylémorphique de la production d'artefacts, héritée d'Aristote : produire, c'est informer, c'est-à-dire doter la matière d'une forme (une idée), comme le giron féminin est rempli par la forme virile (sic). Dans cette perspective, la matière est passive, au mieux résistante. Alors l'homme (dans les deux sens du terme) utilise son arme secrète : l'outil. Face à l'outil, la matière est impuissante. La technique, dans le schéma hylémorphique, est l'auxiliaire de l'information : elle la facilite, elle est purement instrumentale. Elle n'ajoute rien au sens, elle ne fait que l'introduire en douceur dans la matière ${ }^{1}$.

Ce schéma commence à dater et il est toujours majoritaire dans l'esprit des historiens de l'art. Pourtant, quelques grands noms de l'histoire de l'art et de l'esthétique du xxe siècle l'avaient déjà critiqué. Henri Focillon (2000 [1943]), Pierre Francastel (1964), Étienne Souriau (2009 [1943]), Gilbert Simondon (2005 [1954]) en philosophie, pour ne citer que des Français, ont défendu tour à tour l'idée que la matière n'était pas passive, que l'artiste ne devait pas se contraindre à sa forme, mais plutôt qu'il devait composer avec elle : la forme de l'œuvre d'art résulte plus d'un compromis ou d'une alliance entre l'artiste et son matériau, que d'une lutte ou d'une domination. Quant à la technique, loin d'être seulement instrumentale, elle entraîne l'artiste sur des chemins qu'il ne contrôle pas tout à fait. Même si on le manie à la perfection, on ne peut pas tout demander à un outil : celui-ci, pour le dire avec Bruno Latour (2000 : 44), est plein de «promission », mot-valise qui traduit l'affordance de James Gibson : il nous permet de nous en servir de telle ou telle façon, et pas de telle autre, mais il est riche de promesses d'usages inattendus. En anthropologie, Tim Ingold utilise également l'affordance, bien que de manière critique, pour substituer au couple forme/ matière celui des forces et des matériaux, d'inspiration deleuzienne : les objets ne sont plus décrits seulement par leur fonction ou leur ustensilité, mais par leur usage (Ingold 2015 [2013] : 213-214). Ainsi le caillou devient abri pour le crabe qui se réfugie dessous, mais assiette pour le promeneur qui y dépose son sandwich. La forme, intention ou idée, ne rencontre pas un objet-matière neutre, vide de sens : elle surgit plutôt par réaction à l'affordance des choses matérielles.

Le maintien de l'hylémorphisme comme théorie de base de l'histoire de l'art est d'autant plus étrange aujourd'hui que, depuis plus d'une centaine d'années, la définition moderniste de l'art, à savoir faire du médium — du moyen — sa propre fin, s'est, pour sa part, largement imposée. Que l'on définisse le médium artistique tantôt par le support matériel de l'œuvre (la toile du peintre), tantôt par l'outil employé (le film), tantôt par la technique (l'assemblage) importe peu ici puisque dans tous les cas, sa forme ne précède plus, dans l'esprit de l'artiste, sa matérialisation : elle en découle. Et de fait, les rapports aux matériaux, aux outils et aux techniques n'ont cessé de s'enrichir et de se complexifier depuis un siècle. Visiter une exposition d'art contemporain, ce n'est pas tant être confronté à un regard décalé ou critique sur le monde : c'est bien plutôt observer des expériences sur des substances, des usages inattendus d'instruments, des détournements technologiques, qui enrichissent notre relation au concret. Et même si les artistes contemporains ne dialoguent plus avec 
la matière comme le faisaient les peintres ou les sculpteurs de la première moitié du xxe siècle, leur rapport à la technique n'en est que plus riche. Prenons un exemple éclairant. L'artiste Frank Stella a marqué la peinture abstraite au début des années 1960 à New York en imposant des tableaux au dessin géométrique d'une sobriété radicale, qui rompait avec l'expressionnisme de la génération précédente. Faire une peinture originale mais sans aucun épanchement psychologique, telle était son ambition. À partir de 1975 Stella opère apparemment une rupture dans sa propre pratique : il introduit la courbe, des formes biscornues qui s'entremêlent. Les premiers dessins et tableaux de ce nouveau genre s'intitulent Exotic Birds (infra : 126). Les critiques d'art y ont vu, généralement, le besoin qu'éprouvait l'artiste de s'envoler loin du milieu artistique new-yorkais et de renouveler son style sévère par une rupture baroque. Mais le peintre lui-même dit tout autre chose. Il raconte qu'en 1975, il s'achète un jeu de gabarits de dessinateurs appelés aussi perroquets, qui lui donnent l'idée de ces formes courbes (Stella 1986 : 153). De ce point de vue, Stella a changé de formes, mais pas de méthode : il se sert, comme auparavant, d'outils qui lui dictent ses formes : là, la règle et l'équerre; ici, les perroquets. Loin d'être une rupture totale, la série des Exotic Birds développe le travail mené jusqu’alors dans une nouvelle direction. En intégrant ou en négligeant la technique dans l'analyse de l'œuvre, on en change complètement l'interprétation.

Il en va de l'anthropologie comme de l'histoire de l'art. Longtemps et encore dominée par la recherche du sens, la question du « pourquoi ? (Pourquoi les gens accomplissent-ils telles pratiques, développent-ils telles croyances ?), l'anthropologie a cru trouver dans l'analyse des mythes et des structures sociales ses réponses définitives. L'anthropologie des techniques, dans le sillage d'André Leroi-Gourhan, a jeté un regard différent sur les cultures étudiées : le sens ne résidait plus seulement dans les mythes, les rites, les croyances et les structures, mais dans la façon dont les objets étaient fabriqués, dans les techniques du corps, dans le « comment ». Les anthropologues des techniques ont élaboré des concepts et des méthodes d'analyse pour décrire les faits qu'ils étudient, et leur donner un contenu culturel. Si l'histoire de l'art, du fait de son idéologie hylémorphiste, n'est pas bien équipée pour comprendre le rôle des techniques employées par les artistes contemporains, il y a fort à parier que l'anthropologie des techniques pourrait servir de boîte à outils adéquate. Mais il faut tester ces outils pour un usage et sur des matériaux qui ne leur sont pas habituels. Il n'est pas dit qu'ils fonctionnent tous. Sortons cinq outils de la boîte des anthropologues.

\section{I'habileté}

Tim Ingold (2001 : 21-22) estime que le clivage entre art et technique est né de la modernité industrielle et serait donc inadapté pour étudier la fabrication et l'aspect des artefacts produits dans des sociétés non-modernes. La dichotomie art/technique se produit, selon lui, quand l'homme ne se sert plus d'outils mais de machines pour produire des artefacts. Or, si l'outil nécessitait un savoirfaire, une habileté qui engagent la perception, l'intelligence et l'expérience de l'artisan, la machine demande seulement un ouvrier interchangeable capable de l'actionner, sans que le résultat produit 
dépende aucunement de son savoir-faire. Dès lors, la réalisation d'un objet devient une action purement mécanique, totalement distincte de sa conception, qui suppose une connaissance scientifique a priori et rationnelle des lois physiques de la matière détachée de l'expérience de la fabrication même et de ses contingences. C'est ainsi que serait né l'art, par opposition à l'action mécanique et purement technique de l'ouvrier, comme une forme nouvelle d'artisanat. Et effectivement, on peut considérer la peinture, la sculpture, le dessin et autres arts plastiques réalisés à la main, comme des survivances de la production artisanale, où c'est moins l'outil qui est complexe que le savoir-faire qui l'utilise.

De là, une définition en cinq points du savoir-faire ou habileté (skill) par Ingold. 1, l'habileté est une " connaissance pratique et une pratique connaissable ", un savoir-faire qui implique une portée intellectuelle autant que corporelle. 2, l'habileté n'est pas juste une technique du corps, à la Mauss, où le corps serait encore pensé comme un outil passif et mécanique, mais un engagement total de l'être humain, corps et esprit, dans sa perception et son action, son rapport à l'objet et à son environnement. 3, l'habileté n'est pas l'application de la volonté d'un sujet sur un objet, mais un « souci » (care) de cet objet, une façon de le «sentir ». 4, l'habileté ne se transmet pas par écrit ou par des formules figées, mais par la création d'opportunités de perception et d'action. 5, l'habileté n'est pas la correcte mise en œuvre d'une intention mentale, d'une idée ou forme, mais la créativité immanente à l'acte de fabrication même.

Cette riche définition de l'habileté peut être très utile pour étudier les artefacts pré-industriels, qu'ils proviennent de Nouvelle-Guinée ou de l'Italie de la Renaissance. Mais les artistes contemporains utilisent, justement, beaucoup d'objets produits par l'industrie. Ce qui fait art dans leur activité ne se situe pas seulement dans leur habileté manuelle. L'outil « habileté » se révèle donc quelque peu insuffisant, à moins de le redéfinir par-delà l'opposition entre l'artisanat et l'industrie. Si l'on arrive à se dessaisir de l'association, somme toute traditionnelle, entre l'habileté et le savoir-faire manuel, alors le concept d'Ingold peut devenir tout à fait opératoire s'agissant du design et de l'art contemporain : il décrira les façons d'être intensément sensible aux choses, artefacts ou matériaux, et au processus de leur transformation. On pourrait rajouter que ce rapport habile aux choses et aux matériaux définit peut-être une forme de subjectivation propre aux artisans et aux artistes, en extrapolant sur la philosophie de Michel Foucault et les travaux sur la culture matérielle et les processus de subjectivation entrepris par le groupe Matière à penser de Jean-Pierre Warnier et ses collègues (Julien et Rosselin 2009).

\section{I'agentivité}

En anthropologie de l'art, le concept d'agentivité doit presque entièrement sa définition et son application à Alfred Gell, dont le livre posthume, Art and Agency, paru en 1998, continue comme un tremblement de terre de secouer le monde des anthropologues et des théoriciens de l'art (Gell 2009 [1998]). Le concept lui-même, issu de la psychologie cognitive, n'est pas une invention de Gell : il définit la capacité à agir d'un être, humain ou pas, et plus particulièrement, la tel 
que l'emploie Gell, il désigne cette capacité supposée que les humains prêtent aux objets dans certaines conditions. Attribuer à un objet une agentivité, c'est l'envisager comme un membre actif d'une relation sociale. Ou plutôt, l'objet est l'indice d'un agent extérieur ; mais comme cet agent est lointain, invisible ou inconnu, la relation réelle s'effectue avec l'objet qui devient porteur d'agentivité pour lui-même. Ainsi, l'enfant qui joue avec sa poupée comme s'il s'agissait d'un vrai bébé, l'homme pressé qui insulte sa voiture parce qu'elle « refuse », le pauvre malheureux qui incrimine la mine qui lui saute sous les pieds, les fidèles qui vénèrent une statue de la Vierge parce qu'elle « accomplit des miracles » attribuent tous, à leur manière, de l'agentivité aux objets. C'est ce genre de relation, à travers des objets, que Gell nomme art. L'art n'est plus une activité humaine particulière qui concerne un genre particulier d'artefacts (les ouvres d'art des musées), mais le fait qu'on donne aux objets une importance, un statut, qui les assimile à des agents. Résolument anti hylémorphiste, cette théorie permet de prendre au sérieux toutes circonstances dans lesquelles les humains ne considèrent pas le monde qui les entoure comme un réservoir d'objets passifs et inertes. Elle permet aussi d'envisager les œuvres d'art - au sens habituel du terme - sous un angle particulier : elles deviennent les indices d'un agent, en général l'artiste, dont la technique permet à son œuvre de s'autonomiser. Qu'il s'agisse des kolam, ces pas-de-porte ornementaux d'Inde du Sud ou d'un tableau de Vermeer, la technique virtuose de l'artiste impressionne tellement que l'œuvre paraît « enchantée ».

Face aux ready-made de Duchamp cependant, et à beaucoup d'œuvres d'art contemporain dont la virtuosité est la dernière qualité, l'enchantement risque fort d'être de courte durée. Et quand Gell applique sa théorie à une œuvre de Duchamp, il en revient à une forme « classique » d'histoire de l'art. Cela n'en invalide pas pour autant la théorie elle-même et le concept d'agentivité peut être utilisé fertilement pour étudier l'œuvre de certains artistes. Pour n'en citer qu'un, il permettrait de prendre au sérieux l'expression de Joseph Beuys qui parlait de « conversations » entre les objets qu'il installait dans des vitrines préparées à cet effet. S'il y a bien une catégorie de personnes pour qui les objets ou les matériaux ne sont pas inertes mais des partenaires, ce sont les artistes.

\section{Le style}

La notion de style pourrait servir à rapprocher l'histoire de l'art et l'anthropologie des techniques, car dans les disciplines elle a longtemps servi à structurer les discours. On retrouve à chaque fois la même opposition entre deux interprétations du style (Martinelli 2005 : 40). Soit on interprète le style comme un choix opéré dans une chaîne opératoire qui ne change rien quant au résultat fonctionnel attendu, mais qui relève d'une forme d'arbitraire, de gratuité non-fonctionnelle. Un style se reconnaît avant de signifier, il appartient à un niveau cognitif infra-sémiologique, c'est une sorte de sentiment. Soit, au contraire, on interprète le style comme un choix pertinent, dans la chaîne opératoire, en termes de signification culturelle. En somme, 
on retrouve ici l'opposition en histoire de l'art entre le style comme affirmation d'une singularité sur fond de norme, le style comme rupture, comme inconscient corporel ou culturel, et ceci mène au style comme expression de l'individu ou du Kunstwollen d'un peuple, inexplicable mais unique - ce que Leroi-Gourhan appelait pour sa part «l'indéfinissable ethnique »; et le style comme affirmation des traits les plus significatifs d'une culture, précisément ceux qui la représentent le mieux, qui en constituent la norme ; et cela mène à l'iconologie et aux « formes symboliques » de Cassirer (Panofsky 1967 [1939] : 20-21).

Lapproche anthropologique ou iconologique du style suppose aussi toujours son interprétation sociologique, le style comme expression d'un groupe social, ethnique ou pas. Ce qui suppose une sorte de constante stylistique qu'on pourrait résumer à l'équation un peu brutale de l'archéologue Gustav Kossina : un pot, un peuple. On a bien sûr remis en cause cette équation (qu'est-ce qu'un peuple? un même peuple peut adopter plusieurs styles différents dans différents domaines etc.). L'anthropologie structuraliste a mis en valeur les systèmes de transformations qui affectent les formes plutôt que les invariants (Lévi-Strauss sur la peinture caduvéo ou la «split representation », Alfred Gell sur le tatouage dans le Pacifique, entre autres). Reste que ces transformations font système parce que les acteurs qui les accomplissent sont les mêmes ; et que le but des anthropologues, dans ces cas-là, est d'établir des analogies entre ces transformations stylistiques et des transformations sociales. C'est le même type d'analogie qu'Erwin Panofsky établissait entre l'architecture gothique et la scolastique, ou entre la perspective et la rationalité moderne.

Cependant, la notion de style ne nous aidera pas pour aborder une anthropologie des techniques de l'art contemporain; et pour deux raisons. La première, c'est que cette notion n'a plus beaucoup de sens aujourd'hui pour qualifier le travail des artistes. Depuis les années 1960 ceux-ci ont entrepris de détruire tout ce qui pouvait rattacher leur pratique à la question du style. Associé au narcissisme expressionniste de leurs prédécesseurs, le style est devenu gênant à une époque où la phrase de Duchamp — « ce sont les regardeurs qui font les tableaux » — trouvait un écho dans l'affirmation de Barthes — « au règne de l'auteur succède celui du lecteur ». L'artiste ne se conçoit plus comme un être supérieur, un génie, en surplomb au-dessus de la société, mais beaucoup plus comme un passeur, un médiateur, un producteur. À cette conception plus modeste du rôle de l'artiste correspond un idéal utopique de l'art concilié avec la vie.

Et aujourd'hui, si cet idéal n'est plus le credo des jeunes générations d'artistes, il n'en reste pas moins que le style reste une catégorie inopérante d'appréciation de leur travail, puisque chaque artiste contemporain, ou presque, manipule plusieurs médias parallèlement et parfois, le même medium mais de manières différentes. De plus, il est impossible aujourd'hui de définir le style des années 1990, le style des années 2000, tant le nombre d'artistes et la diversité des propositions artistiques ont augmenté - à moins de restreindre l'analyse aux artistes qui ont fait le plus parler d'eux ces années-là. Il suffit d'aller dans une grande biennale d'art contemporain pour se rendre compte de la gageure que constitue le travail de ceux qui cherchent à respirer « l'air du temps » dans ce genre de manifestations. Les artistes n'ont plus de style mais des moyens d'expression qu'ils adaptent selon leurs projets, ou des signatures visuelles qu'ils répètent comme des tampons. 
La seconde raison vient de l'anthropologie des techniques, qui critique aujourd'hui la pertinence de la notion de style. On peut suivre l'exemple de Pierre Lemonnier (Lemonnier 2005 : 250) : celui-ci remarque que le style, chez Leroi-Gourhan, définissait les propriétés marginales d'un artefact, en périphérie des «tendances » lourdes qui sont déterminées par le matériau et contraignent à un nombre de choix techniques restreints. Ainsi, le style (et par extension, l'esthétique, c'est-à-dire les valeurs culturelles propres à chaque peuple) n'est pas directement lié aux choix techniques eux-mêmes. Or, les exemples qu'il donne soulignent que des choix culturels sont déjà présents dans les choix techniques premiers, y compris les choix des matériaux, au cours d'étapes de la chaîne opératoire qui ne se verront même plus forcément dans le résultat final, dans l'aspect extérieur de l'artefact.

\section{La chaîne opératoire}

On tient là un outil peut-être plus efficace que le style. Rappelons que ce concept, inventé par Leroi-Gourhan et popularisé par ses élèves, découle de l'idée qu'un artefact détient une signification culturelle non pas tant par son aspect, ses qualités plastiques, bref son style, que par les choix techniques et matériels qui président à sa fabrication et à son utilisation.

La chaîne opératoire se constitue comme une «syntaxe », elle est nécessaire et obéit à des règles qui échappent en partie à celui qui l'utilise. Autrement dit, l'existence d'une chaîne opératoire marque la présence d'une grammaire technique propre à un groupe social, c'est-àdire l'existence d'une culture. L'individu ne réinvente pas chacun des gestes qu'il accomplit quand il fabrique un objet; cependant, il les maîtrise, il en est conscient, comme quand on se fait un café ou on fait sa toilette. Qu'elle soit «machinale» ou «périodique », l'existence d'une chaîne opératoire articule l'individu au groupe, par le biais de la mémoire collective où elle est entreposée.

Malgré l'idée de « programme » et de « fonds immuable », les chaînes opératoires évoluent, car l'individu joue un rôle actif et conscient dans leur manipulation : il doit s'adapter aux circonstances contingentes, corriger les erreurs, réparer les accidents, et cette intervention est la source de l'innovation (Leroi-Gourhan 1965 : 28-31).

Le travail d'analyse de la chaîne opératoire des artistes n'est quasiment jamais fait par les historiens ou les critiques d'art ; ne serait-ce qu'en cela, ce concept est sans doute l'un des plus utiles à notre entreprise. Mais il faut rajouter que l'articulation de l'individu au groupe, par la chaîne opératoire, n'est plus évidente, dans le cas des artistes modernes et contemporains : leur particularité (par opposition aux artisans ou aux artistes traditionnels) réside en ce qu'ils inventent leur propre chaîne opératoire ; et c'est là, plus que dans leur style, que se tient leur singularité. Grâce à l'emploi de l'outil « chaîne opératoire », on peut comprendre comment une suite d'actions, l'utilisation d'objets, qui, pris isolément, ne sont pas artistiques, permettent de produire pourtant une œuvre d'art. On appellera bricologie l'étude des chaînes opératoires qui 


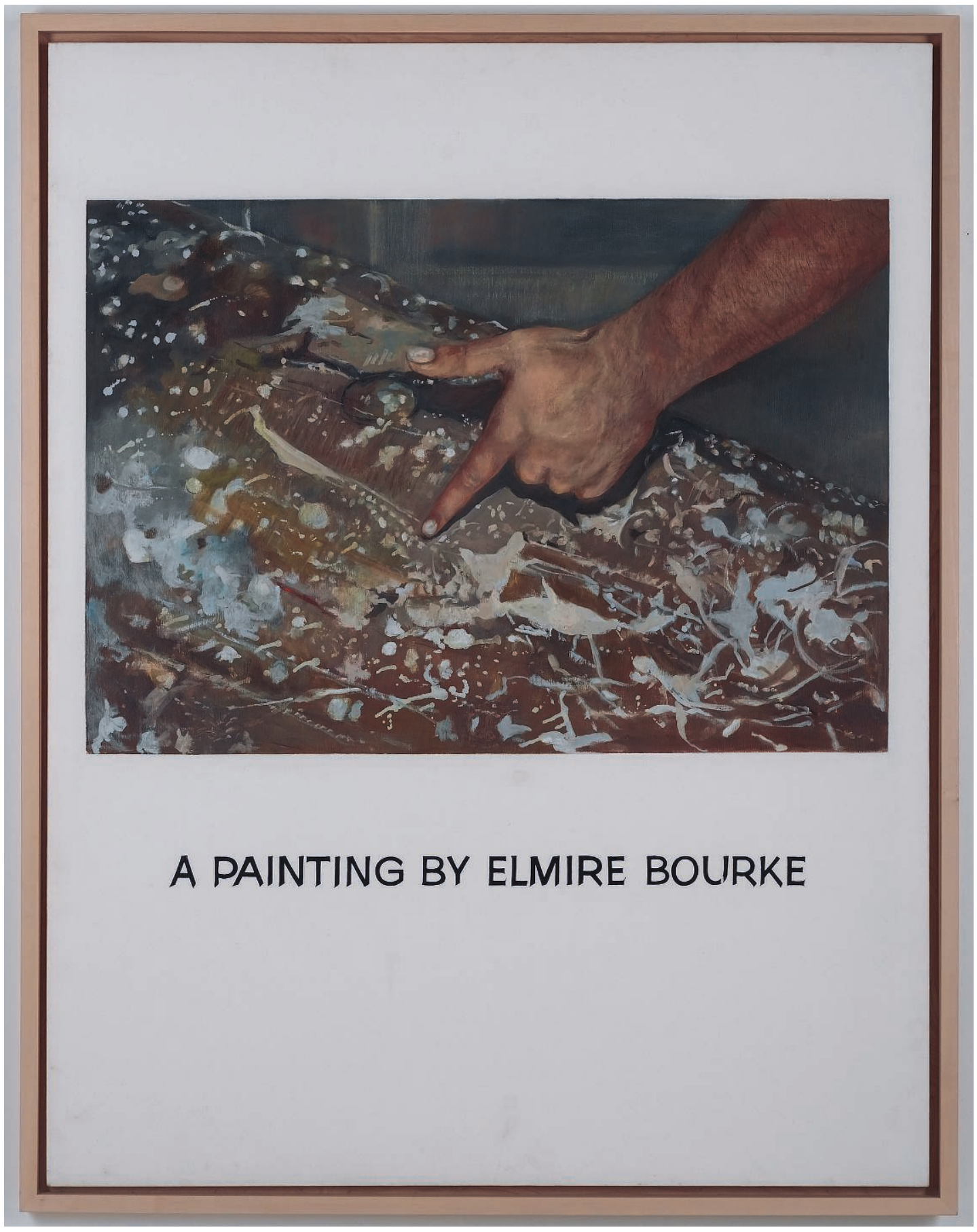

John Baldessari, Commissioned Painting A Painting by Elmire Bourke, 1969. 
mènent à la production d'une œuvre de création contemporaine. Du même coup, certaines postures apparemment anti-techniques, adoptées par les artistes conceptuels, ou par les artistes qui délèguent la production de leurs pièces à des techniciens, peuvent être comprises de manière bricologique. La délégation est l'une des manières les plus utilisées par les artistes pour se débarrasser du style. John Baldessari en constitue un très bon exemple. Dans les années 1960 il a abandonné une peinture expressionniste et paradoxalement peu personnelle, au profit d'une peinture plus froide, constituée de phrases et parfois complétées par des photos transférées sur la toile. D'abord tracées maladroitement par lui, les lettres sont finalement tracées par un peintre lettriste spécialisé. Le tableau se présente ainsi comme vide d'expression manuelle de l'artiste. Mieux : en 1969, il va demander à une quinzaine de peintres amateurs de peindre des tableaux à partir de photos prises par lui, qui représentent toutes un index tendu vers un objet ou une zone sans qualité particulière. Ce qui est remarquable ici, c'est la relation complexe qui s'établit entre l'artiste qui choisit (le geste d'indication en est la figure même) et le peintre qui exécute, mais dont le nom est inscrit en gros, en lieu et place du titre ou de la signature de l'œuvre. De plus, Baldessari s'inspire de la pratique vernaculaire de la «treasure painting » qui consiste à transposer en peinture une photographie personnelle pour la valoriser. La délégation n'est pas simplement le refus de la technique, c'est un choix bricologique qui implique une relation sociale entre au moins deux acteurs, une réflexion sur l'art comme activité professionnelle et comme activité d'amateur, une façon de télescoper la « haute » et la « basse » culture.

\section{Le bricolage}

À tout seigneur tout honneur, terminons par l'outil conceptuel le plus commenté et le plus utilisé par les contributeurs des Essais de bricologie : le bricolage auquel Lévi-Strauss a donné ses lettres de noblesse dans des pages célèbres de La Pensée sauvage (Lévi-Strauss 1990 [1962] : 30-46). Le bricolage peut être envisagé, au même titre que la délégation, comme un certain type de chaîne opératoire artistique, fortement employée aujourd'hui. Je rappellerai juste un point essentiel de cette théorie. Pour Lévi-Strauss, il faut distinguer le bricoleur, l'artiste et l'ingénieur, l'artiste se trouvant pour ainsi dire dans une position intermédiaire entre les deux pôles opposés du bricoleur et de l'ingénieur, entre celui qui récupère tout ce qui peut servir et adapte son projet en fonction de son stock, et celui qui, en bon adepte d'Aristote, part de son projet et trouve les outils et les matériaux dont il a besoin pour le réaliser. L'enjeu de cette distinction, pour Lévi-Strauss, était de montrer une forme de rationalité non-aristotélicienne, non fondée sur des catégories abstraites mais sur l'observation de l'environnement : non pas une pensée de l'abstrait mais du concret. Le bricolage pouvait servir de métaphore préliminaire à la " pensée sauvage » que son livre décrivait ensuite longuement. Cette idée du bricolage comme forme de pensée connut un succès phénoménal ; François Jacob, par exemple, en fit une application remarquée dans le domaine de la biologie de l'évolution (Jacob 1996)². Mais il ne faudrait pas croire que ce n'est 
qu'à titre métaphorique que le bricolage peut évoquer la pensée : si la théorie de Lévi-Strauss a un intérêt à nos yeux, c'est dans la mesure où la pratique effective du bricolage produit de la pensée; en l'occurrence, une pensée de l'invention artistique.

Il est frappant de voir que s'il évoque le Facteur Cheval, la poésie surréaliste et le « hasard objectif » de Breton à propos du bricolage, quand il évoque l'art, c'est à un portrait très classique de Jean Clouet qu'il pense. On sait que Lévi-Strauss n'appréciait pas tellement les arts plastiques de son temps. C'est d'autant plus regrettable qu'il aurait pu y trouver beaucoup de manifestations de sa définition du bricoleur. L'art de la récupération et de l'assemblage, dont le bricoleur fait son beurre, se développe de façon continue depuis les années 1920, avec des pics : les années 1950, et aujourd'hui.

Le bricolage est la pratique artistique qui correspond le mieux, en outre, à la définition de Tim Ingold de l'habileté comme créativité dans l'acte de production lui-même. En effet, contrairement aux artistes qui planifient en amont leur œuvre et demandent à des techniciens de la réaliser, sans autre forme de collaboration ou d'expérimentation, l'artiste bricoleur crée dans l'écart entre son idée de départ et le résultat final. Cet écart est produit par la contingence des matériaux qu'il récupère. Ainsi le bricolage chez un artiste, qui utilise souvent des matériaux pauvres, trouvés dans la rue, n'a rien à voir avec un « mal fait» involontaire, un manque de technique : c'est encore un choix bricologique, celui de faire avec les moyens du bord.

Dans le contexte de crise de la société de consommation, le bricolage constitue, pour l'artiste, un choix autant esthétique qu'éthique et politique. Et c'est ainsi que la singularité des chaînes opératoires mises en œuvre par les artistes peut tout de même acquérir une portée culturelle plus large, raisonner avec les intérêts du groupe d'où ils émergent : plus les artistes se montrent des bricoleurs inventifs et originaux, plus ils constituent une source d'inspiration pour tous les autres. Lartiste bricoleur fait figure de modèle pour tous les adeptes du « do-it-yourself», pour tous ceux qui luttent contre l'obsolescence, programmée ou pas, des produits du commerce, pour tous les « braconniers» de la culture, pour reprendre le terme de Michel de Certeau, qui entendent développer des « tactiques » de résistance face à l'esprit néo-libéral et à la culture de supermarché (de Certeau 1980 [1990]). Une attention bricologique aux œuvres d'art, bien mieux que l'analyse de leur discours, permet d'intégrer pleinement le politique dans les pratiques des artistes, c'est-à-dire de les concevoir comme participant à la vie de la communauté, dont parfois, trop souvent, malgré eux ou avec leur complicité, ils sont exclus.

La boîte à outils de l'ethnologie contemporaine ne contient pas que ces cinq outils. Chaque école, chaque courant développe les siens propres. Parfois, ils sont inopérants quand utilisés sur l'art contemporain. Certains contributeurs aux Essais de bricologie en mobilisent d'autres avec dextérité. Mais il reste encore beaucoup à faire. Nous ouvrons un champ de recherche considérable en espérant que la lecture de ce volume donnera l'envie à d'autres chercheurs de l'explorer à leur tour. 


\section{Notes}

1. Aristote, De la Génération des animaux, livre I, ch. 20,

2. Je remercie Frédéric Joulian de me l’avoir signalé $729 a 10$ - 730a5. Lire aussi Jacobs (1997: 27-33).

\section{I'auteur}

Thomas Golsenne est docteur en histoire de l'art, ancien pensionnaire de l'Académie de France à Rome. Il enseigne actuellement à la Villa Arson, École nationale supérieure d'art de Nice, où il dirige l'unité de recherche Bricologie.

\section{Iconographie}

Image d'ouverture. Peter Hujar, Thek Studio Shoot Thek Working on Tomb Effigy 8, 1967; the Peter Hujar Archive LLC; courtesy Matthew Marks Gallery, New York.

\section{Références}

De Certeau, M. 1990 [1980] L'Invention du quotidien. I, Arts de faire, Paris: Gallimard (Folio).

Flood, R. 1995 [1981] « Paul Thek : une réelle méprise », in The Wonderful World that Almost Was. Paul Thek, catalogue de l'exposition, dir. Roland Groenenboom.

Focillon, H. 2000 [1943] Vie des formes, suivi de Éloge de la main, Paris: PUF

Francastel, P. 1964 Art et technique aux XIXe et XXe siècles, Paris: Denoël

Gell, A 2009 [1998] Art and Agency. An Anthropological Theory, Oxford University Press, trad. fr. S. et $\mathrm{O}$. Renaut, L'Art et ses agents. Une théorie anthropologique, Dijon: Les presses du réel.

Ingold, T. 2015 [2013] « Being Alive to a World Without Objects », in The Handbook of Contemporary Animism, dir. Graham Harvey, Routledge : 213-243.

— 2001 « Beyond Art and Technology : the Anthropology of Skill », in Anthropological Perspectives on Technology, dir. Michael Brian Schiffer, University of New Mexico Press : $17-32$.

Jacob, F. 1996 « Bricolage de l'évolution », in Dictionnaire du darwinisme et de l'évolution, dir. P. Tort, Paris: P.U.F., vol. 1, 414-419.

Jacobs, F. H. 1997 Defining the Renaissance Virtuosa. Women Artists and the Language of Art History and Criticism Cambridge: Cambridge University Press.
1. John Baldessari, Commissioned Painting : A Painting by Elmire Bourke, 1969; Huile et acrylique sur toile 150,5 x 115,6 cm. (C) John Baldessari, courtesy: Ringier Collection, Switzerland.

Julien, M.-P., Rosselin, C (dir.) 2009 Le Sujet contre les objets... tout contre. Ethnographies de cultures matérielles, CTHS, 2009

Latour, B. 2000 « Morale et technique. La fin des moyens » Réseaux, n 100 , vol. 18 : 39-58.

Lemonnier, P. 2005 « Objets sacrés sans "style". Circulez, y’a rien à voir? », in L'Interrogation du style. Anthropologie, technique et esthétique, dir. B. Martinelli, Presses de l'Université de Provence : 237-254.

Leroi-Gourhan, A. 1965 Le Geste et la Parole. II, La Mémoire et les rythmes, Paris: Albin Michel.

Lévi-Strauss, C. 1990 [1962] La Pensée sauvage, Pocket.

Martinelli, B. 2005 «Style, technique et esthétique en anthropologie », in L'Interrogation du style. Anthropologie, technique et esthétique, dir. B. Martinelli, Presses de l'Université de Provence: 19-48.

Panofsky, E. 1967 [1939] Essais d'iconologie. Thèmes humanistes dans l'art de la Renaissance, [trad. fr. C. Herbette et B. Teyssèdre], Paris: Gallimard.

Simondon, G. 2005 [1954] L'Individuation à la lumière des notions de forme et d'information, thèse d'État, Grenoble: Million.

Souriau, E. 2009 [1943] Les Différents modes d'existence, Paris: PUF, rééd. présentée par Isabelle Stengers et Bruno Latour.

Stella, F. 1986 Working Space, Harvard University Press.

\section{Pour citer cet article}

Golsenne, T. 2015 «Les chaînes opératoires artistiques», Techniques\& Culture no 64 «Essais de bricologie. Ethnologie de l'art et du design contemporains», p. 18-31. 
